\title{
Molecular ionization enhancement by charge rearrange- ment at high X-ray intensity
}

Ludger Inhester ${ }^{1,2, *}$, Kota Hanasaki ${ }^{1,2,3}$, Koudai Toyota ${ }^{1,2}$, Yajiang $\mathrm{Hao}^{1,2,4}$, Oriol Vendrell $^{1,2,5}$, Sang-Kil Son ${ }^{1,2,}$, and Robin Santra ${ }^{1,2,6}$

${ }^{1}$ Center for Free-Electron Laser Science, DESY

${ }^{2}$ The Hamburg Centre for Ultrafast Imaging

${ }^{3}$ Department of Chemistry, Graduate School of Science, Tohoku University, Sendai

${ }^{4}$ Department of Physics, University of Science and Technology Beijing

${ }^{5}$ Department of Physics and Astronomy, Aarhus University

${ }^{6}$ Department of Physics, University of Hamburg

Abstract. We simulated the multi-photon multi-ionization dynamics of an iodomethane molecule, $\mathrm{CH}_{3} \mathrm{I}$, exposed to ultraintense and ultrashort $\mathrm{x}$-ray pulses. The strong ionization causes electronic charge rearrangement in the molecule that leads to an enhanced total charge.

\section{Introduction}

The ultraintense and ultrashort x-ray pulses provided by $\mathrm{x}$-ray free-electron lasers can ionize molecules and atoms many times. The theoretical description of these multiple ionization steps is challenging, because many highly excited electronic states are involved in the absorption of $\mathrm{x}$-rays and subsequent decay cascades.

To face this challenge we have developed a new electronic structure toolkit, XMOLECULE, that efficiently describes highly excited and highly charged electronic states[1,2]. We model the X-ray ionization dynamics, by solving multiple coupled rate equations for the populations of the many involved electronic configurations. Within this scheme, the time-dependent population of electronic configuration $I$ is given by

$$
\frac{d}{d t} P_{I}(t)=\sum_{I \neq I^{\prime}} \Gamma_{I^{\prime} \rightarrow I}(t) P_{I^{\prime}}(t)-\Gamma_{I \rightarrow I^{\prime}}(t) P_{I}(t),
$$

where $\Gamma_{I \rightarrow J}(t)$ is the transition rate for an electronic transition from configuration $I$ to $J$, which can be photoionization, Auger decay, or fluorescence. The rates $\Gamma_{I \rightarrow J}(t)$ are computed on-the-fly from electronic structure calculations, which are based on the Hartree-Fock-Slater model $[1,2]$. To efficiently deal with the many involved electronic configurations (trillions for $\mathrm{CH}_{3} \mathrm{I}$ ), we employ the kinetic Monte Carlo technique[3]. Within this scheme, we also propagate the molecular geometry with forces calculated from the electronic structure.

\footnotetext{
*e-mail: ludger.inhester@cfel.de
} 


\section{Results}

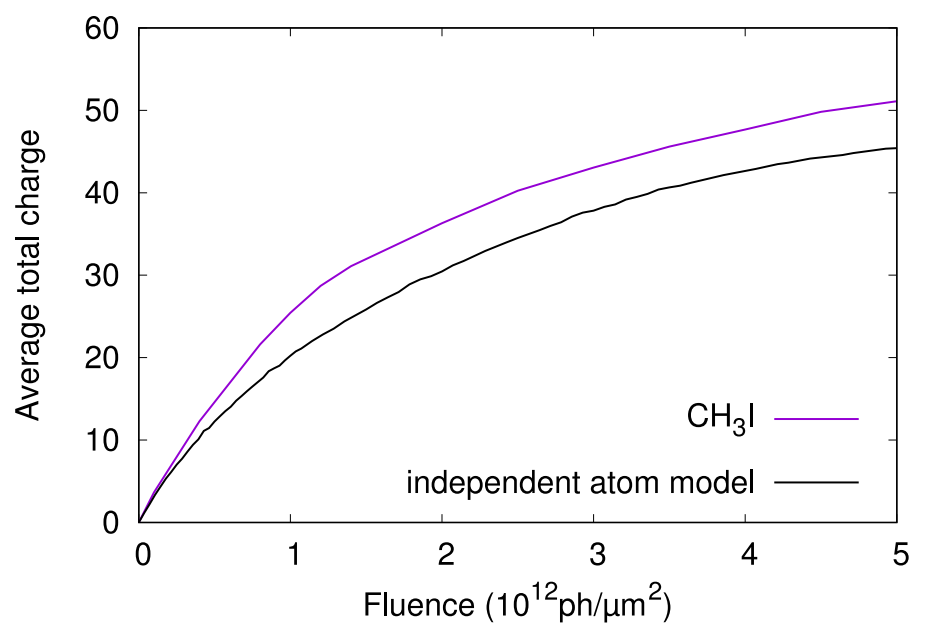

Figure 1. Average total charge as a function of fluence for the molecular calculation and the independent atom calculation.

For an x-ray pulse of photon energy $8.3 \mathrm{keV}$ (Gaussian shaped pulse profile, pulse duration of $30 \mathrm{fs}$ full width half maximum), Fig. 1 compares the total average charge of the molecule after the x-ray pulse as a function of fluence with the independent atom model, in which each atom of the molecule has been simulated independently. Remarkably, the average total charge of the molecule is significantly higher than for the independent atom model[4]. Although the carbon and hydrogen atoms have a negligible cross section for photoionization, their presence leads to an enhanced total charge. This finding demonstrates that electronic rearrangement in molecules can lead to an increase of radiation damage.

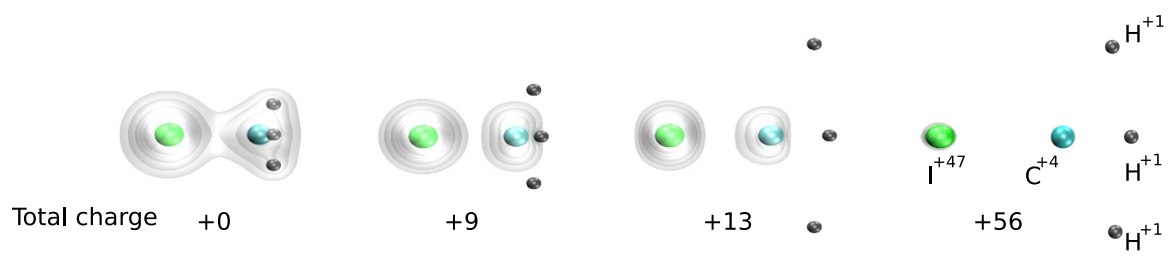

Figure 2. Illustration of the dynamics of a $\mathrm{CH}_{3} \mathrm{I}$ molecule exposed to intense x-ray radiation. Four snapshots at different charge states are shown. The gray cloud indicates the electron density.

The simulation reveals a detailed picture of the break-up of the molecule and the rearrangement of the charges in the molecule. Figure 2 shows four snapshots from a typical simulation at high fluence. We observe that at these high fluences the molecule always dissociates into carbon ions with charge +4 , protons, and highly charge iodine ions.

Our computational model is confirmed by a recent experiment[4], which has measured iodine ion-yields as a function of charge state. To obtain the correct distribution of iodine ion yields, we had to take into account the actual spatial pulse shape, which was determined 


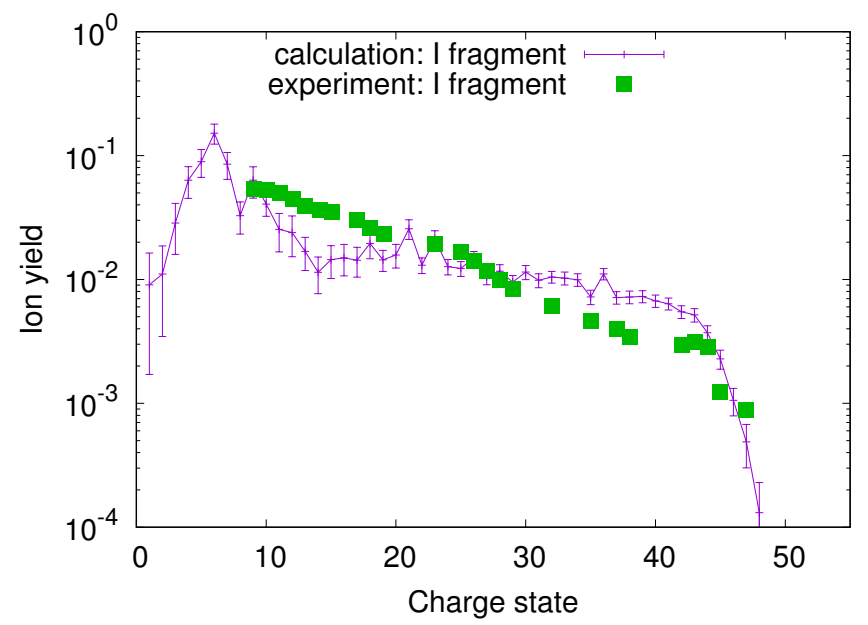

Figure 3. Distribution of iodine ions as a function of charge states. The green dots show experimental data points, whereas the pink curve show calcualted values. The error bars depict the standard error on the ion yields.

by prior calibration measurements. As can be seen in Fig. 3, the calculated and measured iodine ion yield agree well. In particularly, theory and experiment show that iodine ions are produced with charge states up to $q_{I}=47$. At the same time, the experiment confirms via coincidence measurements that high iodine charges $\left(q_{I}>20\right)$ always come together with fully valence-stripped carbon and hydrogen ions $\left(q_{C}=4, q_{H}=1\right)$. These two findings show that the total charge of the molecule is indeed higher compared to what one would expect from isolated atoms as shown in Fig. 1.

Charge rearrangement enhanced ionization dynamics in molecules (CREXIM) can be a crucial factor for assessing the radiation damage in prospective diffractive imaging experiments at these high $\mathrm{x}$-ray intensities, since we expect this effect to be much stronger if more light atoms are present in the surrounding of a strongly absorbing heavy atom.

\section{References}

[1] Y. Hao, L. Inhester, K. Hanasaki, S.K. Son, R. Santra, Struct. Dyn. 2, 041707 (2015)

[2] L. Inhester, K. Hanasaki, Y. Hao, S.K. Son, R. Santra, Phys. Rev. A 94, 023422 (2016)

[3] S.K. Son, R. Santra, Phys. Rev. A 85, 063415 (2012)

[4] A. Rudenko, L. Inhester, K. Hanasaki, X. Li, S.J. Robatjazi, B. Erk, R. Boll, K. Toyota, Y. Hao, O. Vendrell et al., Nature 546, 129 (2017) 